\title{
SECOND-ORDER NEAR-FIELD LOCALIZATION WITH AUTOMATIC PARING OPERATION
}

Rémy Boyer

\author{
Laboratoire des Signaux et Systèmes (L2S) \\ CNRS, Université Paris-Sud XI (UPS), SUPELEC \\ Gif-Sur-Yvette, France \\ remy.boyer@1ss.supelec.fr
}

\author{
José Picheral \\ Département Signaux \& Systèmes \\ SUPELEC \\ Gif-Sur-Yvette, France \\ jose.picheral@supelec.fr
}

\begin{abstract}
Most exiting array signal processing techniques for bearing estimation are strongly relied on the far-field assumption. When the sources are located close to the array, these techniques may no longer perform satisfactorily. In this work, we propose a tensor-based algorithm which is dedicated to the joint estimation of the range and the bearing of multiple narrow-band and near-filed sources in a spatially white Gaussian noise. Automatic paring of the model parameters is achieved for an uniform linear array. By means of numerical simulation, we show that for low Signal To Noise Ratio, the proposed algorithm is more accurate than the Higher Order Statistics (HOS)based ESPRIT algorithm for small/moderate number of snapshots.
\end{abstract}

Index Terms - Parameter estimation, DOA estimation

\section{INTRODUCTION}

An important problem in a wide variety of applications such as radar, sonar, speech, communication, etc. is locating in space the sources of signals received by an array of sensors. Most attention has been restricted to the far-field source model. In the far-field source localization, a source location is characterized only by its bearing since the wavefront from a far-field source can be assumed to be planar for a Uniform Linear Array of sensor (ULA). However, when the sources are located close to the array, the planar assumption is no longer valid. The wavefront in such cases is spherical. In 2D nearfield source localization, a source location is characterized by its bearing and range and the conventional high resolution DOA estimation methods are no longer applicable.

A modified 2D-Music algorithm have been proposed in [1] but it requires a multidimensional search. Higher-Order Statistics (HOS)based methods [2] can handle non gaussian noise and the model parameters pairing is automatic. But these methods are only efficient for a large number of snapshots. A method based on Second-Order Statistics (SOS) has been proposed in [3], it is more accurate than HOS-based methods for small/moderate number of snapshots but it does not provide an automatic paring of the estimates. An SOS-based method with automatic paring has been proposed in [4] but it works only for cross array.

In this paper, we present a tensor-based method exploiting the SOS properties of the model. The interest of this method is that it provides an automatic paring and it has the same accuracy as the SOS-based method.

\section{DEFINITION OF THE MODEL AND ASSOCIATED COVARIANCE SEQUENCES}

\subsection{Definition of the model}

We consider $M$ near-field, uncorrelated, narrowband sources. Each source is characterized by its range $r_{m}$, its bearing $\omega_{m}$ and a complex amplitude $\alpha_{m}(t)$. Let a linear array with $N=2 L+1$ uniformly spaced sensors with interelement spacing $\Delta$. In near-field, using a second-order Taylor expansion, the $t$-th observation on sensor $p \in[-L: L]$ can be approximatively [5] modeled as

$$
x_{p}(t)=\sum_{m=1}^{M} \alpha_{m}(t) e^{i\left(\omega_{m} p+\phi_{m} p^{2}\right)}+n_{p}(t)
$$

where $n_{p}(t)$ is an additive gaussian noise spatially white. The pulsations $\omega_{m}$ and $\phi_{m}$ are functions of the bearing $\theta_{m}$ and the range $r_{m}$ of the $m$-th source, they can be expressed as :

$$
\begin{aligned}
\omega_{m} & =-2 \pi \frac{\Delta}{\lambda} \sin \theta_{m} \\
\phi_{m} & =\pi \frac{\Delta^{2}}{\lambda r_{m}} \cos ^{2} \theta_{m}
\end{aligned}
$$

where $\lambda$ is the source wavelength.

\subsection{Covariance sequences}

If we assume that the near-field sources are uncorrelated, ie., $E\left\{\alpha_{m}(t) \alpha_{m^{\prime}}(t)^{*}\right\}=\sigma_{m}^{2} \delta_{m-m^{\prime}}$ where $\sigma_{m}^{2}$ is the variance of the $m$-th source, then

$$
\begin{aligned}
r_{b}(p) & =E\left\{x_{p+b}(t) x_{p}(t)^{*}\right\} \\
& =\sum_{m=1}^{M} \sigma_{m}^{2} e^{i\left(\omega_{m} b+\phi_{m} b^{2}\right)} e^{i 2 p b \phi_{m}}
\end{aligned}
$$

\section{JOINT VANDERMONDE-TYPE DECOMPOSITION}

\subsection{Definition of the tensor}

Let $\mathcal{H}$ be a $L \times L \times 2$ three-order tensor (or three-way array). We denote by $[\mathcal{H}]_{s}$ the $s$-th vertical slice of tensor $\mathcal{H}$. Then, we define each slice by

$$
[\mathcal{H}]_{1}=H_{0}^{*}, \quad[\mathcal{H}]_{2}=H_{1}
$$


where $*$ means conjugate and

$$
\begin{aligned}
H_{0}= & {\left[\begin{array}{cccc}
r_{-1}\left(-\frac{L}{2}\right) & r_{-1}\left(-\frac{L}{2}+1\right) & \ldots & r_{-1}(0) \\
r_{-1}\left(-\frac{L}{2}+1\right) & r_{-1}\left(-\frac{L}{2}+2\right) & \ldots & r_{-1}(1) \\
\vdots & \vdots & & \vdots \\
r_{-1}(0) & r_{-1}(1) & \ldots & r_{-1}\left(\frac{L}{2}\right)
\end{array}\right], } \\
H_{1} & =\left[\begin{array}{cccc}
r_{1}\left(-\frac{L}{2}\right) & r_{1}\left(-\frac{L}{2}+1\right) & \ldots & r_{1}(0) \\
r_{1}\left(-\frac{L}{2}+1\right) & r_{1}\left(-\frac{L}{2}+2\right) & \ldots & r_{1}(1) \\
\vdots & \vdots & & \vdots \\
r_{1}(0) & r_{1}(1) & \ldots & r_{1}\left(\frac{L}{2}\right)
\end{array}\right]
\end{aligned}
$$

with $r_{b}(p)$ defined in expression (4). Remark that tensor $\mathcal{H}$ is partially Hankel-structured, $i e$., each vertical slice has a Hankel structure.

\section{2. "Two-side" Vandermonde-type decomposition}

First remark that the two covariance sequences involved in each vertical slice is defined according to

$$
\begin{aligned}
r_{1}(p) & =\sum_{m} \sigma_{m}^{2} e^{i\left(\omega_{m}+\phi_{m}\right)} e^{2 i \phi_{m} p} \\
r_{-1}(p) & =\sum_{m} \sigma_{m}^{2} e^{-i\left(\omega_{m}-\phi_{m}\right)} e^{-2 i \phi_{m} p} .
\end{aligned}
$$

Based on these covariance sequences, there exists a "two-side" Vandermonde-structured matrix, defined by

$$
Z=\left[\begin{array}{cccc}
e^{-2 i \phi_{1} \frac{L}{2}} & e^{-2 i \phi_{2} \frac{L}{2}} & \ldots & e^{-2 i \phi_{M} \frac{L}{2}} \\
\vdots & \vdots & & \vdots \\
e^{-2 i \phi_{1}} & e^{-2 i \phi_{2}} & \ldots & e^{-2 i \phi_{M}} \\
1 & 1 & \ldots & 1 \\
e^{2 i \phi_{1}} & e^{2 i \phi_{2}} & \ldots & e^{2 i \phi_{M}} \\
\vdots & \vdots & & \vdots \\
e^{2 i \phi_{1}\left(\frac{L}{2}-1\right)} & e^{2 i \phi_{2}\left(\frac{L}{2}-1\right)} & \ldots & e^{2 i \phi_{M}\left(\frac{L}{2}-1\right)}
\end{array}\right]_{L \times M}
$$

which jointly decomposes each vertical slice of tensor $\mathcal{H}$ according to

$$
\begin{aligned}
H_{0}^{*} & =Z \Theta_{0} Z^{T} \\
H_{1} & =Z \Theta_{1} Z^{T}
\end{aligned}
$$

where

$$
\begin{aligned}
& \Theta_{0}=\left[\begin{array}{ccc}
\sigma_{1}^{2} e^{i\left(\omega_{1}-\phi_{1}\right)} & & 0 \\
& \ddots & \\
0 & & \sigma_{M}^{2} e^{i\left(\omega_{M}-\phi_{M}\right)}
\end{array}\right], \\
& \Theta_{1}=\left[\begin{array}{ccc}
\sigma_{1}^{2} e^{i\left(\omega_{1}+\phi_{1}\right)} & & 0 \\
& \ddots & \\
0 & & \sigma_{M}^{2} e^{i\left(\omega_{M}+\phi_{M}\right)}
\end{array}\right] .
\end{aligned}
$$

\section{ESTIMATION OF THE BEARING AND THE RANGE BY ALS-PARAFAC ANALYSIS}

\subsection{Decomposition of the modes of tensor $\mathcal{H}$}

The particular structure described in the previous section implies a suitable structure into the modes (or also called matrix unfoldings) of tensor $\mathcal{H}$. Let $H^{(1)}, H^{(2)}$ and $H^{(3)}$ be these modes. The explicit definition of these matrices can be found in reference [6] and by tacking into account expressions (10) and (11), it comes

$$
\begin{aligned}
H^{(1)}=H^{(2)} & =\left[\begin{array}{ll}
H_{0}^{*} & H_{1}
\end{array}\right]=Z\left[\begin{array}{ll}
\Theta_{0} Z^{T} & \Theta_{1} Z^{T}
\end{array}\right] \\
& =Z(\Omega \odot Z)^{T} \\
H^{(3)} & =\left[\begin{array}{l}
\operatorname{vec}\left(H_{0}\right)^{H} \\
\operatorname{vec}\left(H_{1}\right)^{T}
\end{array}\right]=\left[\begin{array}{l}
\operatorname{vec}\left(\Theta_{0}\right)^{T}(Z \otimes Z)^{T} \\
\operatorname{vec}\left(\Theta_{1}\right)^{T}(Z \otimes Z)^{T}
\end{array}\right] \\
& =\Omega(Z \odot Z)^{T}
\end{aligned}
$$

where

$\Omega=\left[\begin{array}{llll}\sigma_{1}^{2} e^{i\left(\omega_{1}-\phi_{1}\right)} & \sigma_{2}^{2} e^{i\left(\omega_{2}-\phi_{2}\right)} & \ldots & \sigma_{M}^{2} e^{i\left(\omega_{M}-\phi_{M}\right)} \\ \sigma_{1}^{2} e^{i\left(\omega_{1}+\phi_{1}\right)} & \sigma_{2}^{2} e^{i\left(\omega_{2}+\phi_{2}\right)} & \ldots & \sigma_{M}^{2} e^{i\left(\omega_{M}+\phi_{M}\right)}\end{array}\right]_{2 \times M}$

and $\operatorname{vec}(X)$ is the vector formed by concatenating all the columns of $X, \odot($ resp. $\otimes)$ stands for the Khatri-Rao product (resp. Kronecker product). Note that the first and the second modes are equal since the first and the second sizes of the tensors are also equal $(=L)$.

\subsection{Extraction of the model parameters}

We can extract the model parameters by using the ALS-PARAFAC algorithm [7]. According to expressions (10) and (11), we can see that the $m$-th bearing and the $m$-th range are obtained simultaneously, $i e$, if matrix $Z$ is column-permuted then the diagonal matrices $\Theta_{0}$ or $\Theta_{1}$ are accordingly column-permuted. This structural property explains why the paring operation is automatic. Now, suppose that the resulting matrices of the ALS-PARAFAC analysis are denoted by $Z_{0}, Z_{1}$ and $\bar{\Omega}$. These matrices are known up to a scaling of each of their columns. This corresponds to the indeterminacies in expressions (10) and (11) since there exists two invertible diagonal matrices $D_{0}$ and $D_{1}$ according to

$$
\begin{aligned}
H_{0}^{*} & =Z_{0}\left(D_{0}^{-1} D_{0}\right) \Theta_{0}\left(D_{0} D_{0}^{-1}\right) Z_{0}^{T} \\
H_{1} & =Z_{1}\left(D_{1}^{-1} D_{1}\right) \Theta_{1}\left(D_{1} D_{1}^{-1}\right) Z_{1}^{T} .
\end{aligned}
$$

But, we know that the $(L+1)$-th term of matrices $Z_{0}, Z_{1}$ must be one ( $c f$. matrix $Z$ ) so the scaling operation is characterized by

$$
\hat{Z}=Z_{0} D_{0}^{-1}, \hat{Z}=Z_{1} D_{1}^{-1}
$$

where

$$
\begin{aligned}
& D_{0}=\left[\begin{array}{ccc}
{\left[Z_{0}\right]_{\frac{L}{2}+1,1}} & & 0 \\
& \ddots & \\
0 & & {\left[Z_{0}\right]_{\frac{L}{2}+1, M}}
\end{array}\right], \\
& D_{1}=\left[\begin{array}{ccc}
{\left[Z_{1}\right]_{\frac{L}{2}+1,1}} & & 0 \\
& \ddots & \\
0 & & {\left[Z_{1}\right]_{\frac{L}{2}+1, M}}
\end{array}\right]
\end{aligned}
$$

in which $[Q]_{i j}$ means the $(i, j)$-th entry of matrix $Q$. From expressions (14), (15), (17) and (18), we deduce

$$
\hat{\Omega}=\bar{\Omega} D_{0} D_{1} .
$$

Finally, based on matrices defined in (19) and (22), the model parameter $\left\{\omega_{m}, \phi_{m}\right\}_{m=1, \ldots, M}$ are estimated according to

$$
\left(\hat{\omega}_{m}, \hat{\phi}_{m}\right)=\left(\frac{\angle\left(\frac{[\hat{\Omega}]_{1, m}}{\left[\hat{\Omega}^{*}\right]_{2, m}}\right)}{2}, \frac{1}{L} \sum_{n=1}^{L-1} \angle\left(\frac{[\hat{Z}]_{n+1, m}}{[\hat{Z}]_{n, m}}\right)\right)
$$



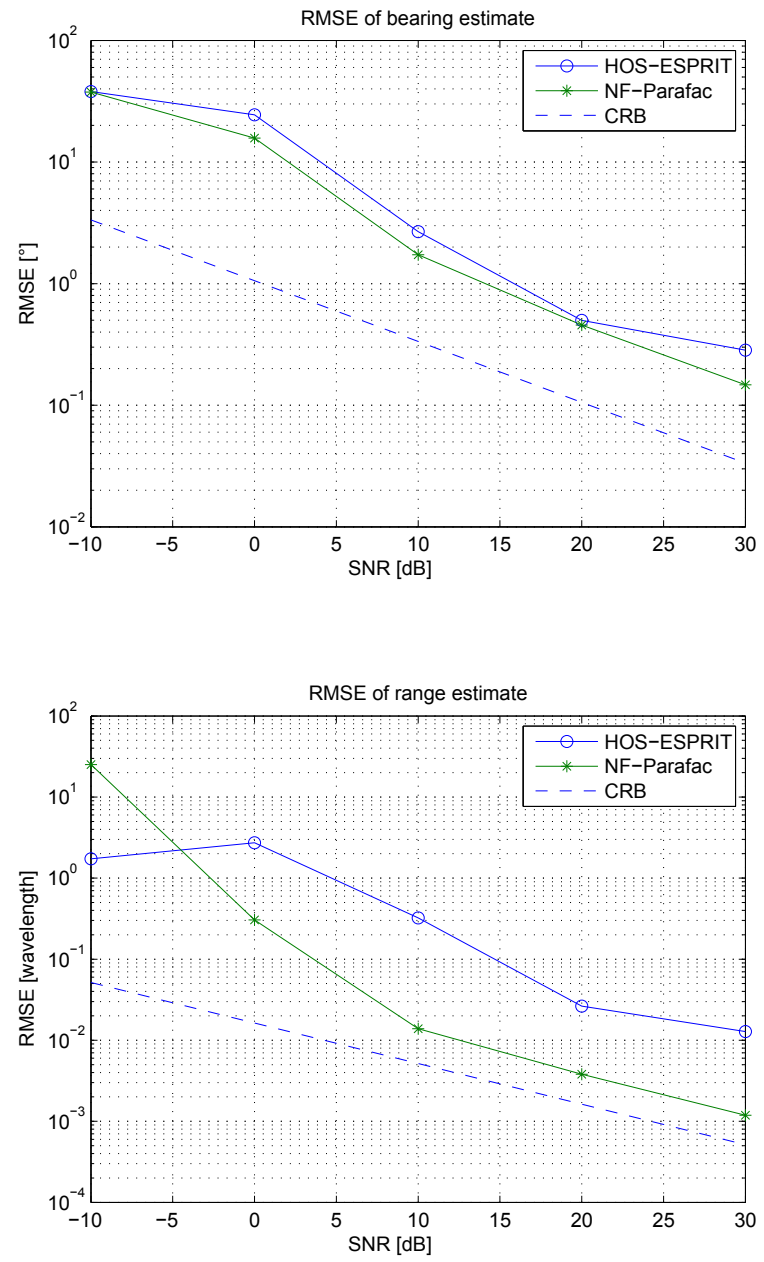

Fig. 1. Example 1 : RMSE of bearing and range estimates vs. SNR for one source with $\theta_{1}=5^{\circ}, r_{1}=0.1 \lambda, 10$ snapshots

where $\angle($.$) is the angle argument. Using (2) and (3), the bearing a$ range parameters can be easily estimated from $\omega_{m}$ and $\phi_{m}$.

Note that as the first and the second modes are identical, we can use a symmetric ALS-PARAFAC model to decrease the complexity burden. In this case, the ALS-PARAFAC algorithm needs only to two pseudo-inverses per iteration instead of three.

\section{SIMULATION RESULTS}

In this simulations we consider an array of $N=17$ sensors with a spacing of Delta $=\lambda / 4$. In order to compare the results with the HOS-ESPRIT method [2], the source signal is not gaussian and is given by $e^{j \xi}$ where $\xi$ is uniformly distributed in $[0,2 \pi]$.

The covariance sequences $r_{-1}(p)$ and $r_{1}(p)$ defined in expression (4) are estimated using the sample covariance estimate :

$$
\begin{aligned}
\hat{r}_{-1}(p) & =\frac{1}{T} \sum_{t=1}^{T} x_{p-1}(t) x_{p}(t)^{*} \\
\hat{r}_{1}(p) & =\frac{1}{T} \sum_{t=1}^{T} x_{p+1}(t) x_{p}(t)^{*}
\end{aligned}
$$
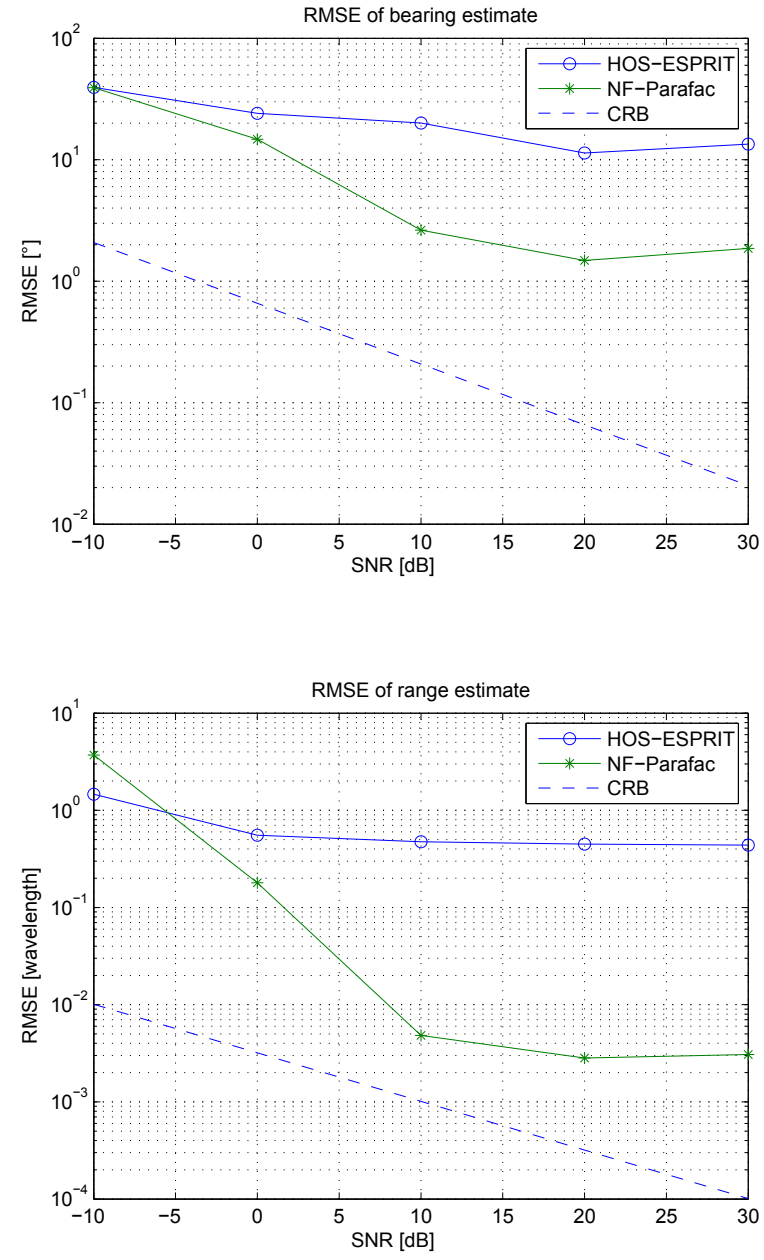

Fig. 2. Example 2 : RMSE of bearing and range estimates vs. SNR for two sources with $\theta=\left[5^{\circ}, 40^{\circ}\right], r=[0.1 \lambda, 0.4 \lambda], 10$ snapshots

where $T$ is the number of snapshot.

\subsection{One source}

In this example, we consider a single source with unit power, bearing is fixed to $\theta_{1}=5^{\circ}$ and range is $r_{1}=0.1 \lambda$. The number of snapshots used to estimate the covariance sequence is 10 . The SNR is varying from $-10 \mathrm{~dB}$ to $30 \mathrm{~dB}$.

On Fig. 1, the Root Mean Square Error (RMSE) of the bearing and range estimate is plotted versus the SNR. Performances of the proposed method (markers $*$ ) are compared with those of the HOS ESPRIT (markers o). In addition, the Cramér-Rao Bound (CRB) is also plotted in dashed line. Expression of CRB can be easily derived for the near-field joint bearing and range estimator (see for instance [8]).

Performances plotted in Fig. 1 show that the RMSE of the proposed method is lower than which of the HOS-ESPRIT method, in particular for the range estimate. Notice also that in this case, the RMSE of the tensor-based method approaches the CRB. 

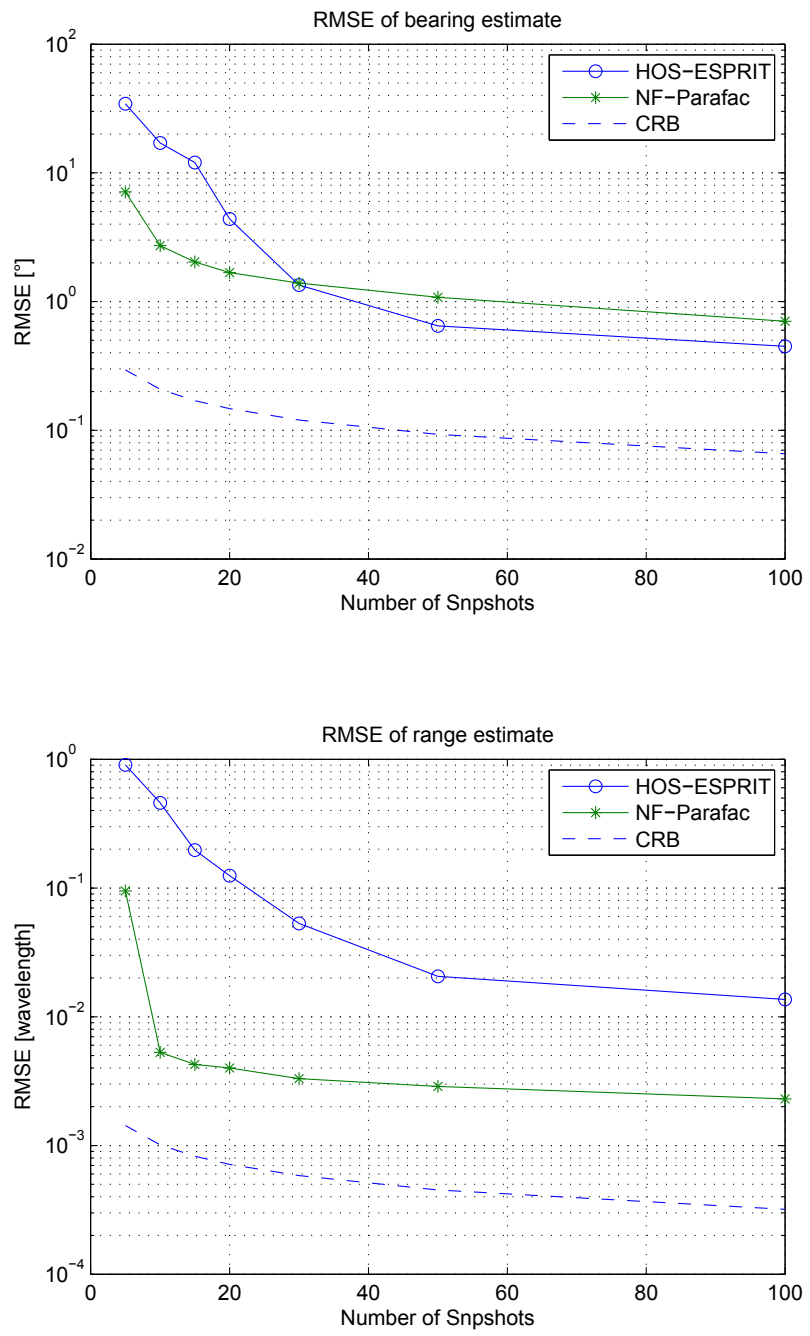

Fig. 3. Example 3 : RMSE of bearing and range estimates vs. the number of snapshots for two sources with $\theta=\left[5^{\circ}, 40^{\circ}\right], r=$ $[0.1 \lambda, 0.4 \lambda], S N R=10 \mathrm{~dB}$

\subsection{Two sources}

In this example, we consider two sources both with unit power, bearing is fixed to $\theta_{1}=5^{\circ}$ and $\theta_{2}=40^{\circ}$, range is $r_{1}=0.4 \lambda$ and $r_{2}=0.1 \lambda$. The number of snapshots is 10 and the SNR is varying from $-10 \mathrm{~dB}$ to $30 \mathrm{~dB}$.

The RMSE plotted in figure 2 show that even if the CRB is not reached, the proposed method achieves an accuracy which is enough for practical applications. Notice that in this case the HOS-ESPRIT method is unable to estimate the bearing and range parameters.

\subsection{Performance with respect to the number of snapshot}

In this example, we consider the same two sources of the previous example, but the SNR is fixed to $10 \mathrm{~dB}$ and the number of snapshots is varying from 5 to 100 .

The RMSE is plotted on figure 3, these simulations show that the interest of the proposed method is mainly for a small or moderate number of snapshots. In this case the HOS methods are known to fail because of the difficulties to estimate cumulant with a small number of snapshots.

All these simulations have shown the advantages of the proposed approach with respect to the HOS-ESPRIT, in particular for small number of snapshots and for low SNR. Notice that these conditions correspond to the situation of interest in practical use of source localization algorithm.

\section{CONCLUSION}

In the context of localization of near-field sources. We propose a new method which exploits a tensor-based scheme of the covariance sequence in order to provide directly an estimation of the pair of bearing and range for each source without an additional pairing operation. Since it is based on the SOS properties of the model, the method is more accurate than the HOS-ESPRIT for small/moderate number of snapshots and in noisy environment. Numerical simulations and comparison with HOS-ESPRIT have demonstrated that the proposed tensor-based algorithm is well suited for a practical application to source localization in near-fields.

\section{REFERENCES}

[1] Y.D. Huang and M.Barkat, "Near-field Multiple Source Localization by Passive Sensor Array", IEEE Trans. Antennas Propag., Vol.39, No.7, 1991, pp.968-974.

[2] N. Yuen, B. Friedlander, "Performance analysis of higher order ESPRIT for localization of near-field sources", IEEE Trans. on Signal Processing, Vol. 46, Issue 3, Mar. 1998, pp : 709-719.

[3] E. Grosicki, K. Abed-Meraim and Y. Hua, ”A weighted linear prediction method for near-field source localization", IEEE Trans. on Signal Processing, Vol. 53, Issue 10, Oct. 2005, pp : 3651-3660.

[4] K. Deng and Q. Yin, "Closed Form Parameters Estimation For 3-D Near Field Sources", Proc. of IEEE International Conference on Acoustics, Speech and Signal Processing (ICASSP'06), May 2006 Vol. 4.

[5] A.L. Swindlehurst, T. Kailath, "Near-Field Source Parameter Estimation Using a Spatial Wigner Distribution Approach", Proc. SPIE Conf., 975, San Diego, CA, August, 1988, pp.8692.

[6] L. De Lathauwer, B. De Moor and J. Vandewalle, "A multilinear singular value decomposition", SIAM J. Matrix Anal. Appl., vol. 21, no. 4, Apr. 2000, pp. 1253-1278.

[7] X. Liu and N. D. Sidiropoulos, "PARAFAC techniques for high-resolution array processing," in Y. Hua, A. Gershman, and Q. Cheng, editors, High Resolution and Robust Signal Processing, Marcel Dekker Inc., New York, pp. 111-150, 2003.

[8] A.J. Weiss, B. Friedlander, "Range and bearing estimation using polynomial rooting", IEEE Journal of Oceanic Engineering, Vol. 18, Apr. 1993, pp : 130-137. 